\title{
Measurement-Induced Entanglement for Excitation Stored in Remote Atomic Ensembles
}

\author{
C. W. Chou, H. de Riedmatten, D. Felinto, S. V. Polyakov, S. J. van Enk, ${ }^{\dagger}$ and H. J. Kimble \\ Norman Bridge Laboratory of Physics 12-33 \\ California Institute of Technology, Pasadena, CA 91125 \\ ${ }^{\dagger}$ Bell Labs, Lucent Technologies, Room 1D-428 \\ 600-700 Mountain Ave, Murray Hill, NJ 07974
}

(Dated: February 1, 2008)

\begin{abstract}
A critical requirement for diverse applications in Quantum Information Science is the capability to disseminate quantum resources over complex quantum networks 1, 2]. For example, the coherent distribution of entangled quantum states together with quantum memory to store these states can enable scalable architectures for quantum computation [3], communication [4], and metrology [5]. As a significant step toward such possibilities, here we report observations of entanglement between two atomic ensembles located in distinct apparatuses on different tables. Quantum interference in the detection of a photon emitted by one of the samples projects the otherwise independent ensembles into an entangled state with one joint excitation stored remotely in $10^{5}$ atoms at each site 6]. After a programmable delay, we confirm entanglement by mapping the state of the atoms to optical fields and by measuring mutual coherences and photon statistics for these fields. We thereby determine a quantitative lower bound for the entanglement of the joint state of the ensembles. Our observations provide a new capability for the distribution and storage of entangled quantum states, including for scalable quantum communication networks [6].
\end{abstract}

Entanglement is a uniquely quantum mechanical property of the correlations among various components of a physical system. Initial demonstrations of entanglement were made for photon pairs from the fluorescence in atomic cascades [7, 8] and from parametric down conversion [9]. More recently, entanglement has been recognized as a critical resource for accomplishing tasks that are otherwise impossible in the classical domain 11. Spectacular advances have been made in the generation of quantum entanglement for diverse physical systems 1, 2], including entanglement stored for many seconds in trapped ions for distances on the millimeter scale 10, 11], long-lived entanglement of macroscopic quantum spins persisting for milliseconds on the centimeter scale 12], and remote entanglement carried by photon pairs over distances of tens of kilometers of optical fibers 13].

For applications in quantum information science, entanglement can be created deterministically by way of precise control of quantum dynamics for a physical system, or probabilistically by way of quantum interference in a suitable measurement with random instances of success. In the latter case, it is essential that success be heralded unambiguously so that the resulting entangled state is available for subsequent utilization. In either case, quantum memory is required to store the entangled states until they are required for the protocol at hand.

There are by now several examples of entanglement generated "on demand," [1] beginning with the realization of the EPR paradox for continuous quantum variables [14] and the deterministic entanglement of the discrete internal states of two trapped ions 15. Important progress has been made towards measurement-induced entanglement on various fronts, including the observation of entanglement between a trapped ion and a photon [16].

Against this backdrop, here we report the initial observation of entanglement created probabilistically from quantum interference in the measurement process, with the resulting entangled state heralded unambiguously and stored in quantum memory for subsequent exploitation. As illustrated in Fig. 1, the detection of a photon from either of two atomic ensembles $(L, R)$ in an indistinguishable fashion results in an entangled state with one "spin" excitation shared at a distance of $2.8 \mathrm{~m}$ between the ensembles and distributed symmetrically among $\sim 10^{5}$ atoms at each site 6]. Confirmation of entanglement is achieved by mapping this stored excitation onto light fields after a $1 \mu$ s delay 6, 17] and by suitable measurements of the quantum statistics of the resulting optical fields. Our results provide the first realization of the capability to transfer a stored entangled state of matter to an entangled state of light.

Our experiment is motivated by the protocol of Duan, Lukin, Cirac and Zoller (DLCZ) 6] for the realization of scalable quantum communication networks with atomic ensembles. The DLCZ protocol introduced a number of novel ideas for quantum information processing and is the subject of active investigation. In this direction, nonclassical correlations [17, 18, 19, 20, 21, 22, 23, 24] and entanglement [25] have been observed between pairs of photons emitted by a single atomic ensemble. Observations of coherence between two cylindrical volumes of cold Rubidium atoms within a single magneto-optical trap have also been reported [26], although entanglement was not demonstrated between the two regions 27,28$]$.

A simple schematic of our experiment is given in Fig. 1, with further details provided in Refs. 17, 21, 23. For the writing stage of the protocol, two classical pulses traverse the $L, R$ ensembles in parallel and generate fields $1_{L}, 1_{R}$ by spontaneous Raman scattering (see Fig. 1a). The intensity of the pulses is made sufficiently weak such that the 
probability of creating more than one excitation in the symmetric collective mode [6] of the ensemble is very low [21].

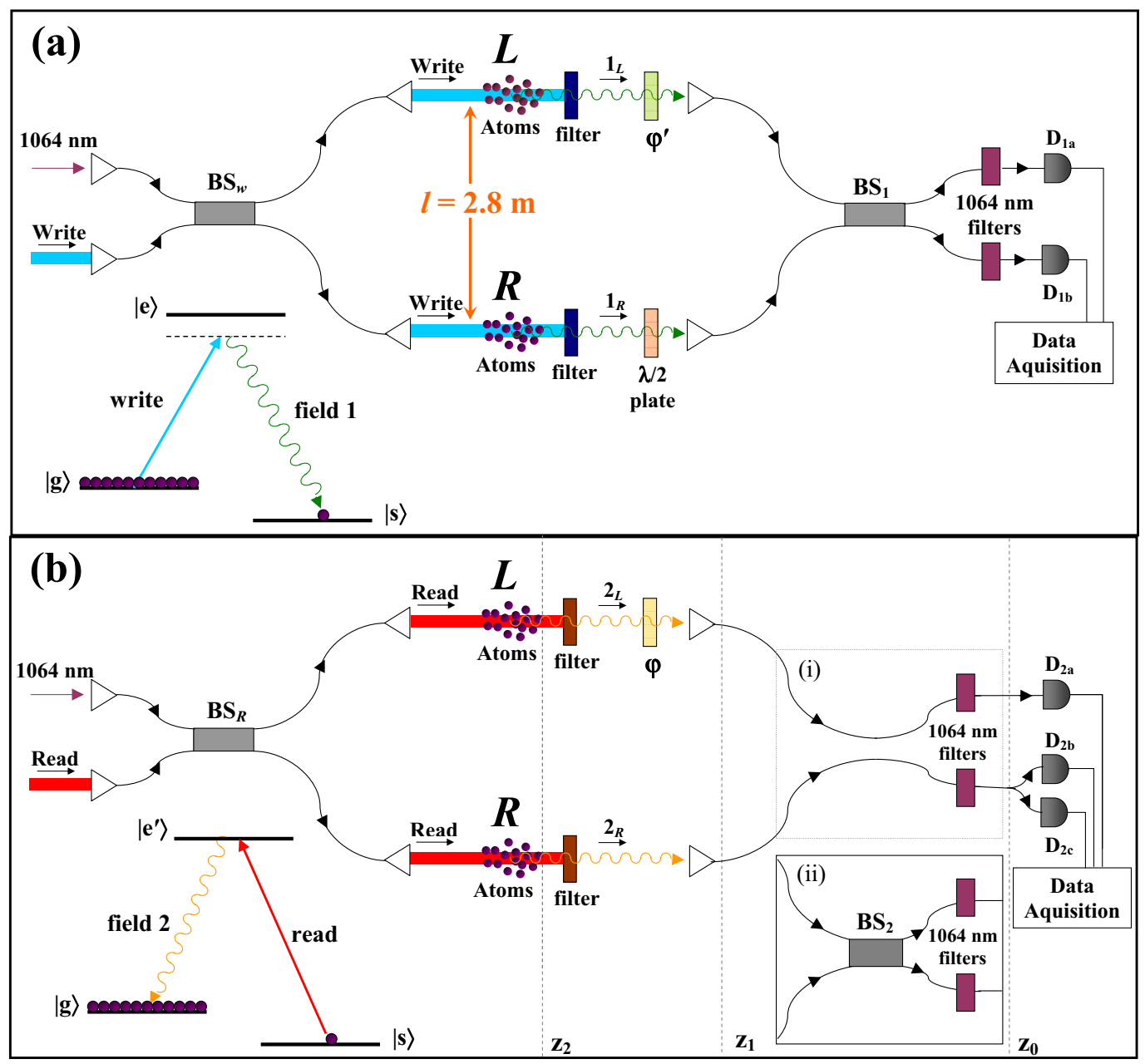

FIG. 1: An overview of our experiment to entangle two atomic ensembles is shown. (a) Setup for generating entanglement between two pencil-shaped ensembles $L$ and $R$ located within spherical clouds of cold Cs atoms. The atomic level structure for the writing process consists in the initial ground state $|g\rangle\left(6 S_{1 / 2}, F=4\right.$ level of atomic Cesium), the ground state $|s\rangle$ for storing a collective spin flip $\left(6 S_{1 / 2}, F=3\right.$ level $)$, and the excited level $|e\rangle\left(6 P_{3 / 2}, F^{\prime}=4\right)$. The transition $|g\rangle \rightarrow|e\rangle$ in each ensemble is initially coupled by a write pulse detuned from resonance to generate the forward-scattered anti-Stokes field 1 from the transition $|e\rangle \rightarrow|s\rangle$. The $L, R$ ensembles are excited by synchronized writing pulses obtained from beam splitter $\mathrm{BS}_{w}$. After filtering, the Stokes fields $1_{L}, 1_{R}$ are collected, coupled to fiber-optic channels, and interfere at beam splitter $B S_{1}$, with outputs directed towards two single-photon detectors $D_{1 a}, D_{1 b}$. (b) Schematic for verification of entanglement between the $L, R$ ensembles by conversion of atomic to field excitation by way of simultaneous read pulses obtained from BS ${ }_{R}$. The read pulses reach the samples after a programmable delay from the write pulses, and couple the transition $|s\rangle \rightarrow\left|e^{\prime}\right\rangle\left(\left|e^{\prime}\right\rangle\right.$ being the $6 P_{1 / 2}, F^{\prime}=4$ level), leading to the emission of the forward-scattered Stokes fields $2_{L}, 2_{R}$ from the transition $\left|e^{\prime}\right\rangle \rightarrow|g\rangle$. The inset (i) shows the configuration used to measure the diagonal elements $p_{i j}$ of $\tilde{\rho}_{2_{L}, 2_{R}}$ in Eq. 3] from the photo-detection events at $D_{2 a}, D_{2 b}, D_{2 c}$. Reconfiguring the fiber connections we can easily pass from the configuration of inset (i) to the one of inset (ii), which is used to generate interference of the $2_{L}, 2_{R}$ fields at beam splitter $B S_{2}$ to measure the off-diagonal coherence $d$ in $\tilde{\rho}_{2_{L}, 2_{R}}$. In (a) and (b), the incident write and read beams are orthogonally polarized and combined at a polarizing beam splitter, and are focused to a waist of about $30 \mu \mathrm{m}$ in the sample region. All beam splitters BS are polarization maintaining fiber beam splitters. The $\approx 12 \mathrm{~m}$ arms of both write and read interferometers are actively stabilized using an auxiliary Nd:YAG laser at $1.06 \mu \mathrm{m}$.

Entanglement between the $L, R$ ensembles is created by combining the output fields $1_{L}, 1_{R}$ on the beam splitter $B S_{1}$, with outputs directed to two photodetectors $D_{1 a}, D_{1 b}$ (see Fig.1a). For small excitation probability and with unit overlap of the fields at $B S_{1}$, a detection event at $D_{1 a}$ or $D_{1 b}$ arises indistinguishably from either field $1_{L}$ or $1_{R}$, so that the $L, R$ ensembles are projected into an entangled state, which in the ideal case can be written as 6 , 29]

$$
\left|\Psi_{L, R}\right\rangle=\epsilon_{L}|0\rangle_{L}|1\rangle_{R} \pm e^{i \eta_{1}} \epsilon_{R}|1\rangle_{L}|0\rangle_{R}
$$


where $|0\rangle_{L, R},|1\rangle_{L, R}$ refers to the two ensembles $L, R$ with 0,1 collective excitations respectively, $\epsilon_{L}\left(\epsilon_{R}\right)$ is the normalized amplitude of photon generation from ensemble $L(R)$, and the sign \pm is set by whichever detector records the event. The phase $\eta_{1}=\Delta \beta_{w}+\Delta \gamma_{1}$, where $\Delta \beta_{w}$ is the phase difference of the write beams at the $L, R$ ensembles, and $\Delta \gamma_{1}$ is the phase difference acquired by the $1_{L}, 1_{R}$ fields in propagation from the ensembles to the beam splitter $B S_{1}$. Note that to achieve entanglement as in Eq. 1 $\eta_{1}$ has to be kept constant from trial to trial.

To verify the entanglement, we map the delocalized atomic excitation into a field state by applying simultaneously strong read beams at the two ensembles (see Fig.1b). If the state transfer were to succeed with unit probability, the conditional state $\left|\Psi_{L, R}\right\rangle$ of the ensembles would be mapped to an entangled state of two modes for the Stokes fields $2_{L}, 2_{R}$ given in the ideal case by [6, 29]

$$
\left|\Phi_{L R}\right\rangle=\epsilon_{L}|1\rangle_{2_{L}}|0\rangle_{2_{R}} \pm e^{i\left(\eta_{1}+\eta_{2}\right)} \epsilon_{R}|0\rangle_{2_{L}}|1\rangle_{2_{R}}
$$

where $|0\rangle_{2_{L}, 2_{R}},|1\rangle_{2_{L}, 2_{R}}$ refers to the Raman fields $2_{L}, 2_{R}$ with 0,1 photons, respectively. Here, $\eta_{2}=\Delta \beta_{r}+\Delta \gamma_{2}$, where $\Delta \beta_{r}$ is the phase difference of the read beams at the $L, R$ ensembles, and $\Delta \gamma_{2}$ is the phase difference acquired by the $2_{L}, 2_{R}$ fields in propagation from the ensembles to the beam splitter $B S_{2}$ in Fig. 1b. In our experiment, the phases $\eta_{1}, \eta_{2}$ can be independently controlled and are actively stabilized by utilizing auxiliary fields at $1.06 \mu \mathrm{m}$ that copropagate along the paths of the write and read beams and of the $1_{L}, 1_{R}$ and $2_{L}, 2_{R}$ fields.

Of course, the states in Eqs. 12 are idealizations that must be generalized to describe our actual experiment 6, 27, 29]. Specifically, the presence of various sources of noise necessarily transforms these pure states into mixed states. Eqs. 10 also neglect the vacuum contribution as well as higher-order terms, which are intrinsic to DLCZ protocol and which otherwise arise due to diverse experimental imperfections. Moreover, the above analysis assumes that all excitations are in the correct "modes" (both for optical fields and for the collective atomic "spin flips"), that excitations of the ensembles map 1-to-1 to photons in fields 1 and 2, and that diverse sources of background light are absent.

The procedure that we have devised to provide a robust, model independent determination of entanglement is based upon quantum tomography of the $2_{L}, 2_{R}$ fields (see Appendix B for details). Since entanglement cannot be increased by local operations on either of the two ensembles, the entanglement for the state of the ensembles will be always greater than or equal to that measured for the state of the light fields. Specifically, conditioned upon a detection at $D_{1 a}$ or $D_{1 b}$, we consider the density matrix

$$
\tilde{\rho}_{2_{L}, 2_{R}}=\frac{1}{\tilde{P}}\left(\begin{array}{cccc}
p_{00} & 0 & 0 & 0 \\
0 & p_{01} & d & 0 \\
0 & d^{*} & p_{10} & 0 \\
0 & 0 & 0 & p_{11}
\end{array}\right)
$$

which is written in the basis $|n\rangle_{2_{L}}|m\rangle_{2_{R}}$, with the number of photons $\{n, m\}=\{0,1\} . p_{i j}$ is then the probability to find $i$ photons in mode $2 L$ and $j$ photons in mode $2 R$, and $d$ gives the coherence between the $|1\rangle_{2_{L}}|0\rangle_{2_{R}}$ and $|0\rangle_{2_{L}}|1\rangle_{2_{R}}$ states. $\tilde{\rho}_{2_{L}, 2_{R}}$ is obtained from the full density matrix $\rho_{2_{L}, 2_{R}}$ by restricting it to the subspace where there is at most one photon in each mode, with then $\tilde{P}=p_{00}+p_{01}+p_{10}+p_{11}$. Since the concurrence $C\left(\tilde{\rho}_{2_{L}, 2_{R}}\right)$ for $\tilde{\rho}_{2_{L}, 2_{R}}$ provides a lower bound for the concurrence $C\left(\rho_{2_{L}, 2_{R}}\right)$ for $\rho_{2_{L}, 2_{R}}\left[C\left(\rho_{2_{L}, 2_{R}}\right) \geq \tilde{P} C\left(\tilde{\rho}_{2_{L}, 2_{R}}\right)\right]$, we devise measurements to deduce the various components of $\tilde{\rho}_{2_{L}, 2_{R}}$. The concurrence $C\left(\tilde{\rho}_{2_{L}, 2_{R}}\right)$ can then be calculated from Eq. B] by way of Ref. [30],

$$
\tilde{P} C=\max \left(2|d|-2 \sqrt{\left(p_{00} p_{11}\right)}, 0\right) .
$$

The entanglement of formation $E$ follows directly from $C$, where $E$ and $C$ both range from 0 to 1 for our system and $E$ is a monotonically increasing function of $C[30]$.

\begin{tabular}{|ccc|}
\hline Probability & $D_{1 a}$ & $D_{1 b}$ \\
\hline$p_{00}$ & $0.98510 \pm 0.00007$ & $0.98501 \pm 0.00007$ \\
$p_{10}$ & $(7.38 \pm 0.05) \times 10^{-3}$ & $(6.19 \pm 0.04) \times 10^{-3}$ \\
$p_{01}$ & $(7.51 \pm 0.05) \times 10^{-3}$ & $(8.78 \pm 0.05) \times 10^{-3}$ \\
$p_{11}$ & $(1.7 \pm 0.2) \times 10^{-5}$ & $(1.9 \pm 0.2) \times 10^{-5}$ \\
\hline
\end{tabular}

TABLE I: Diagonal elements of the density matrix $\tilde{\rho}_{2_{L}, 2_{R}}$ [Eq. (3)], deduced from the records of photo-electric counts. The values of $p_{i j}$ are referenced to the location of detectors $D_{2 a, 2 b, 2 c}$, and were obtained by considering unit detection efficiency, which gives a more conservative (i.e., smaller) lower bound for the concurrence than the actual (larger) field concurrence for finite efficiency $<1$. See Appendix A for further details. 
As a first step in the determination of $C$ we measure the diagonal elements $p_{i j}$. As shown in Fig. 1b, the field-2 output of each ensemble is directed to different sets of detectors in order to record photon counting probabilities for the fields $2_{L}, 2_{R}$ separately. From the record of photoelectric counting events, we then deduce the diagonal elements of $\tilde{\rho}_{2_{L}, 2_{R}}$, which are listed in Table 1. From Eq. 团and noting that $|d|^{2} \leq p_{10} p_{01}$, a necessary requirement for $C>0$ is that there be a suppression of two-photon events relative to the square of the probability for single-photon events for the fields $2_{L}, 2_{R}$ i.e., $h_{c}^{(2)} \equiv p_{11} /\left(p_{10} p_{01}\right)<1$. For our measurements, we find $h_{c}^{(2)}=0.30 \pm 0.04$ for events conditioned on detection at $D_{1 a}$, and $h_{c}^{(2)}=0.35 \pm 0.04$ for events conditioned on $D_{1 b}$ [21]. In contrast, for non-conditioned events, we find $h_{n c}^{(2)}=0.99 \pm 0.04$.

The second step in our tomography protocol is to determine the coherence term $d$ in Eq. 3 which we accomplish by adding a relative phase shift $\varphi$ for the fields $2_{L}, 2_{R}$, and by combining them at the beam splitter $B S_{2}$ shown in Fig. 1b. By recording the conditional count rate after the beam splitter as function of $\varphi$, one can measure an interference fringe with a visibility $V$, with then $|d|$ following from $V$ and the $p_{i j}$. Roughly, for $50 / 50$ beam splitters and neglecting higher order terms (that are employed in our actual analysis), we would have $|d| \cong V\left(p_{10}+p_{01}\right) / 2$.

Figure 2 shows conditional counts $N_{2 a}, N_{2 b}+N_{2 c}$ as functions of $\varphi$. These data demonstrate that the indistinguishable character of measurement events at detectors $D_{1 a}$ (Fig.2a) and $D_{1 b}$ (Fig.2b) induces a high degree of coherence between the otherwise independent ensembles $L, R\left[\underline{6}\right.$, 26] . Indeed, we deduce visibilities $V_{1 a}=(70 \pm 2) \%$ and $V_{1 b}=(71 \pm 2) \%$ for the associated conditional states.

A notable feature of these results is that the interference fringes have relative phase $\pi$ for the cases of detection at $D_{1 a}, D_{1 b}$, in agreement with Eqs. 10 2] We observe similar fringes if the phase $\eta_{1}$ between the write beams is varied instead of $\varphi$. Moreover, if the fields $1_{L}, 1_{R}$ are combined at the beamsplitter $B S_{1}$ with orthogonal polarizations (by way of the half-wave plate in Fig. 1a), we find that the visibility from interference of fields $2_{L}, 2_{R}$ drops to near zero, since in this case, there is no longer measurement-induced entanglement associated with quantum interference for detection of fields $1_{L}, 1_{R}$ (see Appendix A).

With Eq. [4 the measured values for the visibility $V$ and for the various $p_{i j}$ are sufficient to deduce a lower bound for the concurrence $C$ for the field state $\tilde{\rho}_{2_{L}, 2_{R}}$ at the location of detectors $D_{2 a, 2 b, 2 c}$, with no correction for detection efficiencies or propagation losses, we find

$$
C_{1 a}\left(\tilde{\rho}_{2_{L}, 2_{R}}\right)=(2.4 \pm 0.6) \times 10^{-3}>0, C_{1 b}\left(\tilde{\rho}_{2_{L}, 2_{R}}\right)=(1.9 \pm 0.6) \times 10^{-3}>0,
$$

conditioned upon detection at either $D_{1 a}$ or $D_{1 b}$. This conclusively demonstrates a nonzero degree of entanglement between the ensembles, albeit with the concurrence $C_{L, R}$ small. The small difference between the concurrence for the states conditioned on $D_{1 a}$ or $D_{1 b}$ can be explained by an asymmetry in $B S_{w}$ (see Appendix A).

Beyond the firm lower bound given by Eq. 5] we can make a better estimate of the degree of entanglement $C_{L, R}$ between the $L, R$ ensembles by way of detailed measurements of the propagation efficiencies from the atomic ensembles to the plane $z_{0}$ of the detectors shown in Fig. 1b (see Appendix A). Figure 3 gives an inference of the density matrix $\tilde{\rho}_{2_{L}, 2_{R}}^{z_{i}}$ and thereby of the concurrence $C^{z_{i}}\left(\tilde{\rho}_{2_{L}, 2_{R}}^{z_{i}}\right)$ at $z_{0}$ and at two other locations $z_{i=1,2}$ along the path from the ensembles to the detectors (see Fig. 1b), assuming a constant visibility. Generally, $C$ increases in direct correspondence to the reduced level of losses for the $2_{L}, 2_{R}$ fields at locations closer to the ensembles. At location $z_{2}$ corresponding to the output edges of the atomic ensembles, we find the result

$$
C_{L, R}^{1 a} \geq C_{1 a}^{z_{2}}\left(\tilde{\rho}_{2_{L}, 2_{R}}^{z_{2}}\right) \simeq 0.021 \pm 0.006>0, C_{L, R}^{1 b} \geq C_{1 b}^{z_{2}}\left(\tilde{\rho}_{2_{L}, 2_{R}}^{z_{2}}\right) \simeq 0.016 \pm 0.006>0 .
$$

To move beyond this result, we need more detailed information about the efficiencies $\xi_{L, R}$ with which stored excitation in the atomic ensembles is converted to the propagating light fields $2_{L}, 2_{R}$. Our earlier measurements included comparisons to a simple model [21] and allowed an inference $\xi_{L, R} \sim 0.10 \pm 0.05$. The measurement of the losses together with the values of $p_{i j}$ at the detectors yields $p_{10}+p_{01} \approx 11 \%$ at the output of the ensembles $\left(z_{2}\right.$ plane) for our current experiment. This value together with the estimated $\xi_{L, R}$ then indicates that $p_{00} \rightarrow 0$ for the conditional state $\rho_{L, R}$ of the ensembles, so that $C_{L, R} \approx V \approx 0.7$, suggesting that $\rho_{L, R}$ is close to the ideal entangled state of Eq. 1] The low measured values for the entanglement between fields $2_{L}, 2_{R}$ apparently are principally a consequence of the low readout efficiency $\xi_{L, R}$ of the atomic excitation. We stress that this inference of $C$ for the state inside the ensembles must be confirmed by subsequent experiments and is offered here to provide some insight into future prospects for quantum protocols with entangled ensembles. This also emphasizes that a central point in subsequent work should be the improvement of $\xi_{L, R}$.

In conclusion, we have achieved entanglement between a pair of atomic ensembles separated by $2.8 \mathrm{~m}$, with the entangled state involving one spin excitation within a collective system of roughly $10^{5}$ atoms at each site $L, R$. The entangled state is generated by and conditioned upon an initial detection event, and is thus produced in a probabilistic fashion. However, this initial event heralds unambiguously the creation of an entangled state between $L, R$ ensembles, which is physically available for subsequent utilization, as, for example, by mapping to propagating optical fields, which can be in principle accomplished with high efficiency. We emphasize that our measurements relate to an actual 


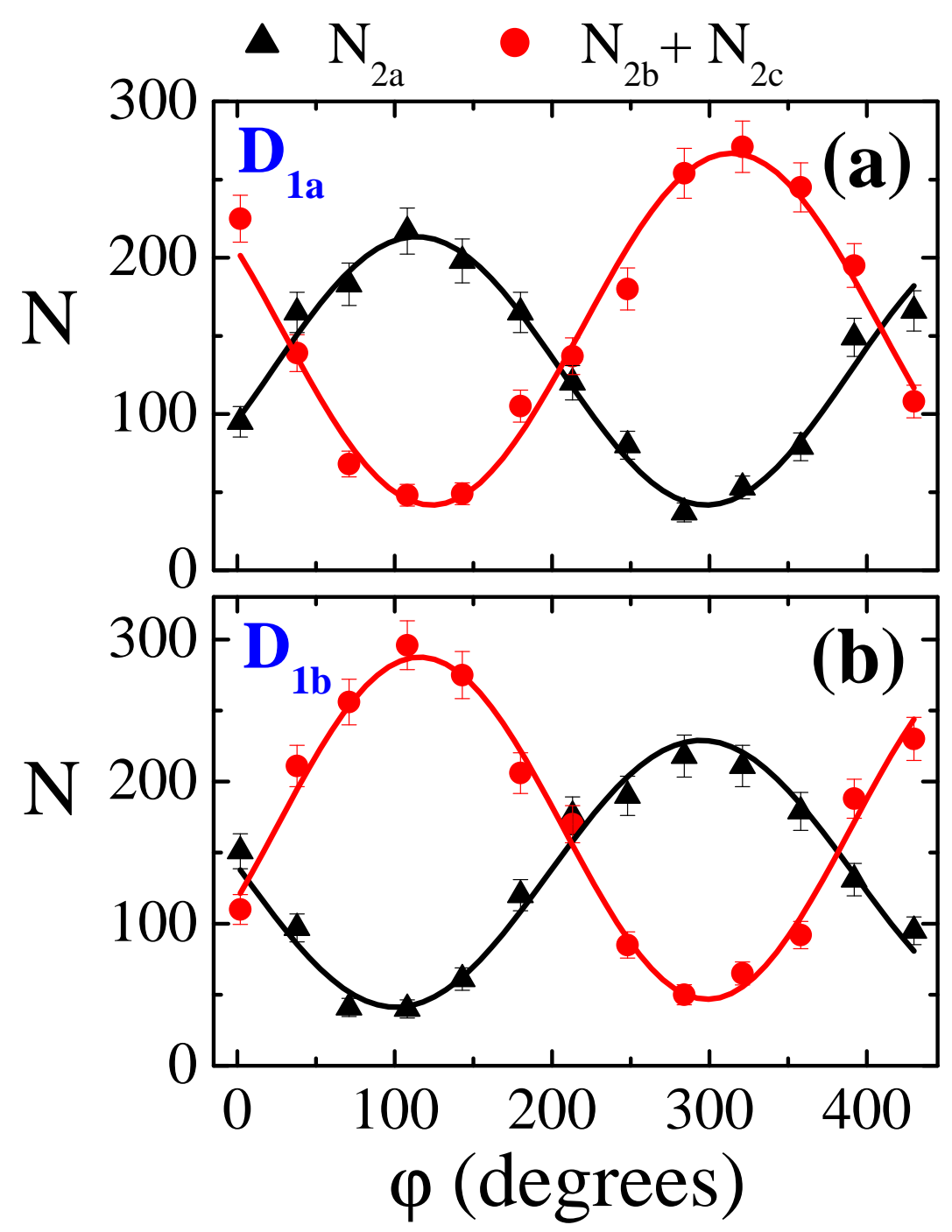

FIG. 2: Coherence between the atomic ensembles $L, R$ is induced by a measurement event of the fields $1_{L}, 1_{R}$ at detector $D_{1 a}$ or $D_{1 b}$. Shown is the number of coincidences $N_{2 a}$ (circles) and $N_{2 b}+N_{2 c}$ (triangles) recorded by the respective detectors $D_{2 a, 2 b, 2 c}$ for the fields $2_{L}, 2_{R}$ with the interferometer arrangement of Fig. $1 \mathrm{~b}$ as a function of the relative phase $\varphi$. In (a) $N_{2 a, 2 b, 2 c}$ are conditioned upon a detection event at $D_{1 a}$ with no count at $D_{1 b}$, while in (b) $N_{2 a, 2 b, 2 c}$ are conditioned upon an event at $D_{1 b}$ with no count at $D_{1 a}$. At each setting of $\varphi$, data are acquired for $150 \mathrm{~s}$ with a detection window of width $190 \mathrm{~ns}$. Although the interference fringes have comparable visibility, the different sizes arise from unequal quantum efficiencies for detectors $D_{2 a}$ and $D_{2 b, 2 c}$ (see Appendix A). The visibility values are obtained from an average of the visibilities of the red and black curves, respectively. Error bars reflect \pm one standard deviation due to the finite number of counts.

physical state of the $L, R$ ensembles and of the $2_{L}, 2_{R}$ fields, and are not an inference of a state based upon post selection. Our work provides the first example of a stored atomic entangled state that can be transfered to entangled light fields, and significantly extends laboratory capabilities for entanglement generation, with now entangled states of matter stored with separation a hundred-fold larger than was heretofore possible for continuous quantum variables and a thousand-fold larger than for qubits. With our current setup, we have demonstrated $\Delta t_{s} \simeq 1 \mu \mathrm{s}$ for storing entanglement. However, this should be readily extended to $\Delta t_{s} \simeq 10 \mu \mathrm{s}$, and new trapping schemes have the potential to lead to $\Delta t_{s} \simeq 1 \mathrm{~s}$ [17]. The distance scale for separating the $L, R$ ensembles is limited by the length $l_{0} \simeq 2 \mathrm{~km}$ for fiber optic attenuation at our write wavelength $852 \mathrm{~nm}$. Extensions to scalable quantum networks over larger distances requires the realization of a quantum repeater [6], for which we have now laid the essential foundation. 
(a)

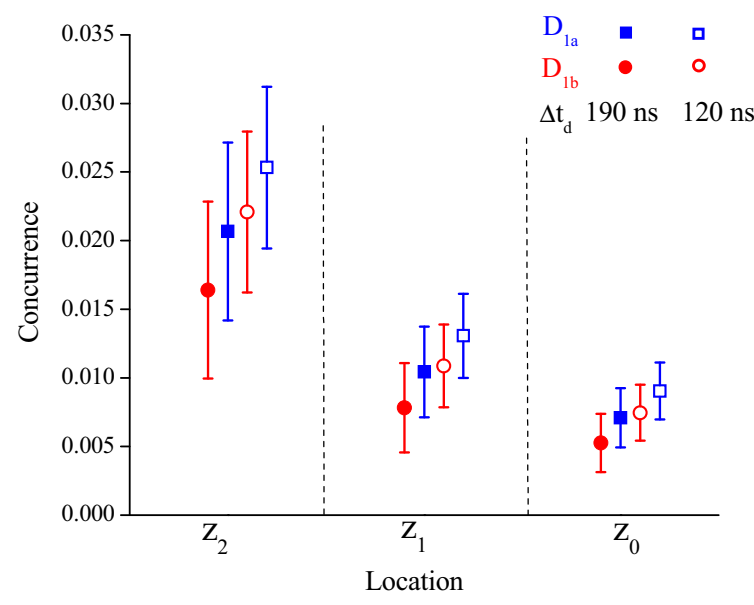

(b)
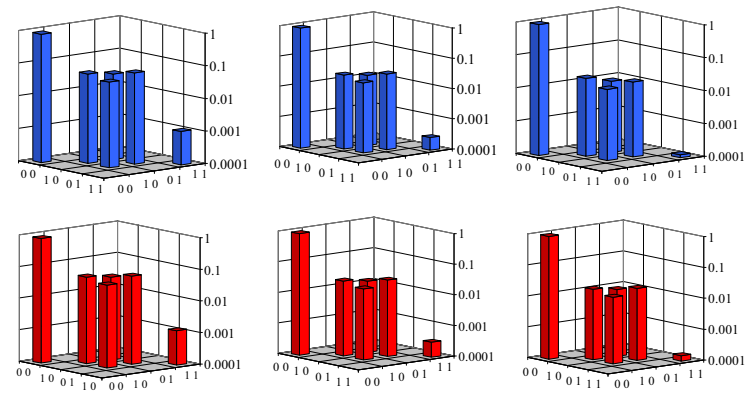

$\mathrm{Z}_{2}$

$\mathrm{Z}_{1}$

$\mathrm{Z}_{0}$

Location

FIG. 3: The results of our measurements for the concurrence $C^{z_{i}}$ (a) and density matrix $\tilde{\rho}_{2_{L}, 2_{R}}^{z_{i}}$ (b) are shown at the three locations $z_{i}$ indicated in Fig. 1b. At each location, two pairs of results are given corresponding to the measurement-induced state created from detection at $D_{1 a}$ and at $D_{1 b}$, and taking into account the efficiency of the detectors and propagation losses. (a) Concurrence $C$, for two different detection windows $\Delta t_{d}$ at $D_{2 a, 2 b, 2 c}$. Filled symbols are for $\Delta t_{d}=190 \mathrm{~ns}$, enough to acquire the whole temporal wavepacket of field 2. Open symbols are for $\Delta t_{d}=120 \mathrm{~ns}$. We see then that the degree of entanglement can be further enhanced, similar to the increase of nonclassical correlations between fields 1 and 2 reported in Ref. 23] for specific detection windows for these fields. All values shown in this figure, including the ones for $z_{0}$, are already corrected for the efficiencies of the detectors. Error bars reflect \pm 1 standard deviation, taking into account the finite number of counts and the uncertainties in the efficiency and propagation loss. (b) Density matrix $\tilde{\rho}_{2_{L}, 2_{R}}^{z_{i}}$ given in the basis $|n\rangle_{2_{L}}|m\rangle_{2_{R}}$ corresponding to Eq. 3] with $\{n, m\}=\{0,1\}$ and $\Delta t_{d}=190 \mathrm{~ns}$.

Acknowledgement - This research is supported by the Advanced Research and Development Activity (ARDA), by the National Science Foundation, and by the Caltech MURI Center for Quantum Networks. D.F. acknowledges financial support by CNPq (Brazilian agency). H.d.R. acknowledges financial support by the Swiss National Science Foundation. SJvE thanks Lorenz Huelsbergen for his assistance in computer matters.

\section{APPENDIX A: EXPERIMENTAL DETAILS}

\section{Atomic ensembles and optical pulses}

Each of the $L, R$ atomic ensembles is obtained from Cesium atoms in a magneto-optical trap (MOT) 17, 21]. Measurements are carried out in a cyclic fashion consisting first of a period of cooling and trapping to form the MOT, 
followed by an interval during which the magnetic fields for the MOT are switched off. After waiting $3 \mathrm{~ms}$ for the magnetic field to decay [17], we initiate a sequence of measurement trials, where for each trial the atoms are initially prepared in level $|g\rangle$. The write pulse is at $852 \mathrm{~nm}$, with a duration of $150 \mathrm{~ns}$ and is detuned $10 \mathrm{MHz}$ below the $|g\rangle \rightarrow|e\rangle$ transition. The read pulse is at $894 \mathrm{~nm}$, with a duration of $130 \mathrm{~ns}$ and is resonant with the $|s\rangle \rightarrow\left|e^{\prime}\right\rangle$ transition. At the end of each trial, the sample is pumped back to level $|g\rangle$ by illuminating the atomic cloud with trapping and repumping light for $0.7 \mu \mathrm{s}$ and $1 \mu \mathrm{s}$ respectively, and then a new trial is initiated with period of $3 \mu \mathrm{s}$. The total duration for a sequence of measurement trials is $5 \mathrm{~ms}$, after which the measurement interval is terminated and a new MOT is formed in preparation for the next sequence of trials.

\section{Losses and efficiencies}

In order to infer the concurrence of the fields at different locations in our experimental apparatus it is important to characterize the losses in the different components of the communication channel of field 2. Our measurements for such losses are given in the table below for the pathways starting from each of the atomic ensembles. Immediately after the ensembles, we separate the classical pulses from fields 1 and 2 in the single-photon level. This procedure is explained in detail in Refs. 18, 21]. The losses coming from this stage are due mainly to passage through paraffin coated vapor cells, which have transmission $\alpha_{f c}$. After the filtering process, field 2 is coupled to a polarization maintaining fiber that carries it to the detection region. The coupling efficiency is denoted by $\alpha_{c}$. In the detection region, it is important first to filter the $1.06 \mu \mathrm{m}$ light that propagates together with field 2, and which is used to actively lock the read interferometer when $B S_{2}$ is inserted. In order to filter the $1.06 \mu \mathrm{m}$ light, field 2 comes out of the fiber and pass trough a bulk low-pass filter that reflects $1.06 \mu \mathrm{m}$ light and transmits with high efficiency at $894 \mathrm{~nm}$. At this stage, we also include an extra filter for $852 \mathrm{~nm}$, to cut any residual light from the write process, and then field 2 is coupled again to a fiber, this time a multi-mode one. This whole process of passing through the band-pass filters and recoupling to fiber is called generally "filter for $1064 \mathrm{~nm}$ ", and is characterized by a transmission $\alpha_{f}$. Finally, the detector efficiencies are given by $\alpha_{A P D}$. Note that a single number is given for the pair of detectors $D_{2 b, 2 c}$, since they both have measured efficiencies of $40 \%$.

\begin{tabular}{|ccccc|}
\hline Description & Symbol & Value for ensemble L & Value for ensemble R & Error \\
\hline \hline filter cell & $\alpha_{f c}$ & 0.80 & 0.80 & 0.02 \\
fiber coupling & $\alpha_{c}$ & 0.70 & 0.65 & 0.02 \\
1064 nm filter & $\alpha_{f}$ & 0.70 & 0.70 & 0.02 \\
detector & $\alpha_{A P D}$ & 0.32 & 0.40 & 0.02 \\
Total & $\alpha$ & 0.13 & 0.15 & \\
\hline
\end{tabular}

TABLE 1: List of efficiencies associated with photon 2 propagation and detection.

\section{Suppression of interference between the $2_{L}, 2_{R}$ fields for distinguishable detection events from the fields $1_{L}, 1_{R}$}

In our experiment, entanglement is generated by quantum interference between the fields 1 emitted by the ensembles, that are combined at a beam-splitter. For this interference to occur, the two fields must be indistinguishable, such that no information can be obtained about the origin of the photons. A good way to illustrate the importance of this overlap is to render the photons distinguishable, for instance by combining the two fields 1 with orthogonal polarizations. In this way, information about the origin of the detected photon is near maximal, and the degree of measurement-induced entanglement should be significantly reduced (to zero in the ideal case). The results of such a measurement are shown in Fig. 4. Fig. 4(a,b) shows the interference fringes obtained when the photons of field 1 are combined with parallel polarizations (same fringes as in Fig. 2), while in Fig. 4(c,d), the photons from field 1 are combined with orthogonal polarizations. In the latter case, the visibility drops to near zero, and there is no entanglement between the two ensembles. The residual oscillation in the conditional count rate can be explained by the finite polarization extinction ratio in our polarization maintaining fibers. The fibers used in our experiment have a measured extinction ratio of $28 \mathrm{~dB}$ between their two orthogonal propagation modes. This can lead to a residual visibility of $8 \%$, which is compatible with the amplitude of the residual oscillation in Fig. 4(c,d). 


\section{Asymmetry in the creation of the states conditioned on $D_{1 a}$ and $D_{1 b}$}

The difference in the two sets of probabilities $\left(p_{01}^{(1 a)}, p_{10}^{(1 a)}\right)$ and $\left(p_{01}^{(1 b)}, p_{10}^{(1 b)}\right)$ results from an asymmetry in the beam splitter $B S_{1}$ for detection of the write fields $1_{L}, 1_{R}$, with a measured ratio of transmission to reflection $T / R=0.85$. Hence, in addition to the \pm sign in Eq. 1 of set by detection at $D_{1 a}$ or $D_{1 b}$, the relative amplitudes for the conditional state can also differ, resulting in different values for the concurrence. We expect the ratio $\left(p_{01}^{(1 a)} / p_{10}^{(1 a)}\right)\left(p_{01}^{(1 b)} / p_{10}^{(1 b)}\right)^{-1}$ to be $(T / R)^{2}=0.73$, which agrees well with the measured value $(7.51 / 7.38)(8.78 / 6.19)^{-1}=0.72$.

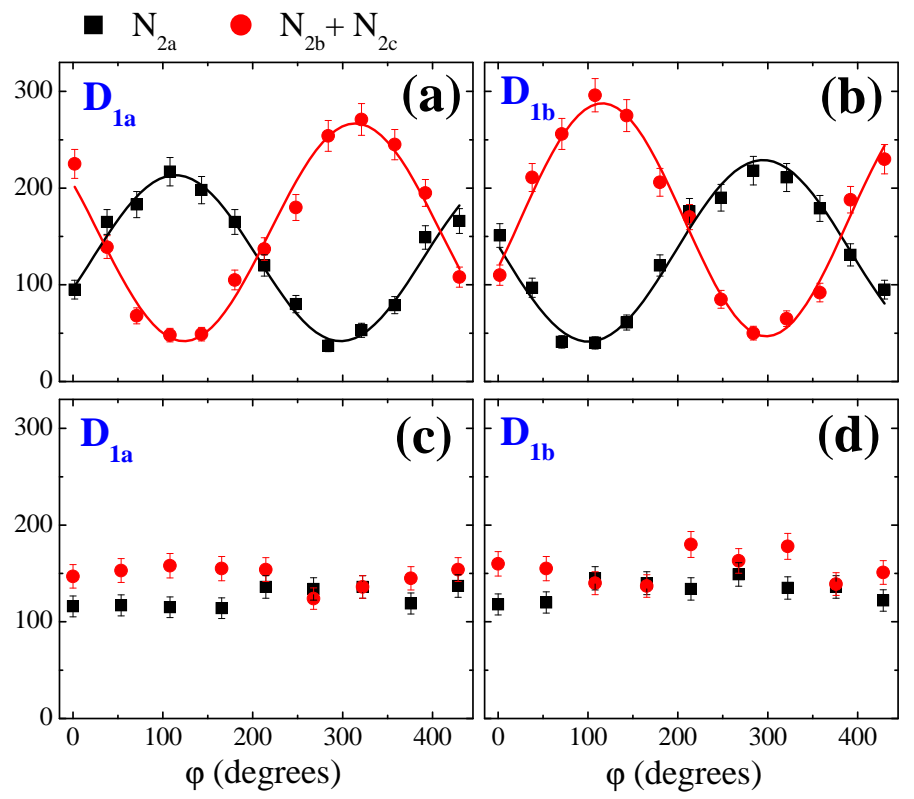

FIG. 4: Number of coincidences $N_{2 a}$ (squares) and $N_{2 b}+N_{2 c}$ (circles) recorded by the respective detectors $D_{2 a, 2 b, 2 c}$ for the fields $2_{L}, 2_{R}$ with the interferometer arrangement of Fig. 1(b) as a function of the relative phase $\varphi$. Frames (a) and (b) are the same as frames (a) and (b) of Fig. 2, i.e., they show the interference fringe between fields $2_{L}, 2_{R}$ as a result of combining fields $1_{L}, 1_{R}$ in an approximately indistinguishable fashion with parallel polarizations. Frames (c) and (d) show the results of the same measurement on fields $2_{L}, 2_{R}$, but now with fields $1_{L}, 1_{R}$ combined with orthogonal polarizations. At each setting of $\varphi$, data are acquired for $150 \mathrm{~s}$ with a detection window of width $190 \mathrm{~ns}$.

\section{Verification of single excitation regime}

Apart from the parameter $h_{c}^{(2)}$ discussed previously, another measure of the single-photon character of field 2 is given by the evaluation of the function $\tilde{g}_{12}=\tilde{p}_{12} /\left(\tilde{p}_{1} \tilde{p}_{2}\right)$ for each ensemble separately [18, 21], where $\tilde{p}_{12}$ is the joint probability of detecting a photon in field 1 and another in field 2 , and $\tilde{p}_{i}$ is the probability of detecting a single photon in field $i$. For the situation of our measurement, we obtained $\tilde{g}_{12}$ values of about 9 and 11 for ensembles $R$ and $L$, respectively, which is then an indication of the single-photon character of field 2 emitted by both ensembles separately, as discussed in [21].

\section{Phase stabilization.}

The $\approx 12 \mathrm{~m}$ path lengths for the two interferometers formed by $\left(B S_{w}, B S_{1}\right)$ and by $\left(B S_{R}, B S_{2}\right)$ shown in Figures 1a,b, respectively, are held constant by injecting an iodine stabilized Nd:YAG laser into the fiber beam splitters $B S_{w}, B S_{R}$ for the write and read beams. The fields at $1.064 \mu \mathrm{m}$ emerging from beam splitters $B S_{1}, B S_{2}$ are directed to separate sets of detectors (not shown in the figure), whose outputs are utilized to stabilize the relative path lengths of the respective Mach-Zehnder interferometers by feedback to piezoelectric transducers on which are mounted mirrors in the paths of the write and read beams in the $L$ arms of the respective interferometers. Since the write and read beams are combined together before being focused into the ensembles, the interferometers share a common free space path 
when they cross the atoms. In order to control them independently and minimize crosstalk, the two interferometers are addressed alternately at a rate of $400 \mathrm{kHz}$.

\section{Normalization and detector configuration}

The role of the three detectors in our experiment is as a second check on the order of magnitude of the two-photon events. Our main checks were discussed in the manuscript and in section A5 of this Appendix. It is interesting to obtain directly $p_{02}$ and have an idea of its effect on $p_{01}$. For our measurement, we obtained, with $D_{2 b}$ and $D_{2 c}$, $p_{02}=(2.2 \pm 0.4) \times 10^{-5}$ conditioned on detector $D_{1 a}$, and $p_{02}=(2.4 \pm 0.4) \times 10^{-5}$ conditioned on detector $D_{1 b}$. Note that these values are so small that if taken into account, the correction for $p_{10}$ and $p_{01}$ would be negligible. In order to simplify our analysis and inversion algorithm, we consider the three detectors as an effective set of two detectors. This is done by simply adding the coincidence events between $D_{2 b}$ and $D_{2 c}$ to the sum $N_{2 b}+N_{2 c}$, in the same way that would result from the use of a non-number-resolving detector. In this case, the measured normalization constant $\tilde{P}$ is equal to one, since all measured events contribute to one of the elements of the restricted density matrix. If we had used more detectors, the discrepancy of $\tilde{P}$ from unity would be on the order of $p_{02}$ and negligible compared to the experiment accuracy.

\section{APPENDIX B: THEORY}

\section{Entanglement}

For convenience of description we assume the two atomic ensembles $L$ and $R$ to be in the hands of Alice and Bob, respectively. The state of the two ensembles conditioned on a click of one of the two detectors $D_{1 a}$ or $D_{1 b}$ (see Figure $1 \mathrm{~b}$ of main text) is mapped onto a state of multiple field modes belonging to Alice and multiple modes belonging to Bob. Because the mapping involves only local operations by Alice and Bob, the entanglement (in particular, the entanglement of formation) between their systems cannot increase on average 31]. Hence the entanglement found between Alice's and Bob's field modes is a lower bound on the entanglement between the atomic ensembles. We will use this type of reasoning several times here: certain experimental procedures can be exactly mimicked by imagining Alice and Bob performing LOCC (local operations and classical communication): those operations can only decrease the entanglement we find. We also sometimes (lower) bound the entanglement analytically using quantities that are more straightforward to measure in the laboratory. That way, we can unambiguously determine the presence of entanglement between the two ensembles, even if we might underestimate its actual magnitude.

On each side there is one main mode (a traveling continuous-wave mode) into which photons are emitted predominantly []. . Those modes we denote by $2_{L}$ and $2_{R}$. Other modes may be populated with very small probability, but in the analysis we assume all detector clicks arise from modes $2_{L}$ and $2_{R}$. In the experiment this reduction from multiple to single modes is mainly accomplished by the use of single mode fibers, which filter out different spatial modes. This is a procedure that can be exactly mimicked by Alice and Bob performing that same spatial filtering on their local modes and hence can only decrease the actual entanglement.

We also assume that never more than 2 photons populate each mode. This is an excellent approximation on its own (and is supported by our measurements), but more importantly, this assumption corresponds to lower bounding the entanglement, as detailed below.

We furthermore assume that all off-diagonal elements of the density matrix between states with different numbers of photons vanish. This is a valid assumption when one takes into account that that phase can only be defined relative to a reference laser beam shared by Alice and Bob. Tracing out that laser field sets the off-diagonal elements to zero. Indeed, the experiment makes no use of knowledge of the phases of the various lasers used. Moreover, this can only underestimate the entanglement, since tracing out the laser modes can be exactly mimicked by Alice and Bob performing local operations that makes all those off-diagonal elements zero. Namely, they could each apply a random phase shift to their modes, such that the phase difference is fixed (this requires classical but not quantum communication), and subsequently ignore the information about the individual phase shifts. The phase difference is equal to the phase $\varphi$ introduced in the main text. 
This then leaves us with a density matrix of the form

$$
\rho_{2_{L}, 2_{R}}=\left(\begin{array}{cccccc}
p_{00} & 0 & 0 & 0 & 0 & 0 \\
0 & p_{01} & d & 0 & 0 & 0 \\
0 & d^{*} & p_{10} & 0 & 0 & 0 \\
0 & 0 & 0 & p_{11} & e & f \\
0 & 0 & 0 & e^{*} & p_{02} & g \\
0 & 0 & 0 & f^{*} & g^{*} & p_{20}
\end{array}\right) .
$$

We can bound the entanglement of formation of this state by

$$
E\left(\rho_{A, B}\right) \geq \tilde{P} E\left(\tilde{\rho}_{2_{L}, 2_{R}}\right)
$$

where

$$
\begin{aligned}
\tilde{P} & =p_{00}+p_{01}+p_{10}+p_{11} \\
\tilde{\rho}_{2_{L}, 2_{R}} & =\frac{1}{\tilde{P}}\left(\begin{array}{cccc}
p_{00} & 0 & 0 & 0 \\
0 & p_{01} & d & 0 \\
0 & d^{*} & p_{10} & 0 \\
0 & 0 & 0 & p_{11}
\end{array}\right)
\end{aligned}
$$

One obtains this bound by considering the effects of two local operations by Alice and Bob consisting of measuring whether each mode has more than 1 photon or not and communicating this result one to the other. We treat this step explicitly and separately from the very similar step mentioned above [where cases with more than two photons are filtered out], in order to remind ourselves we do have to keep track of the total probability to find more than 1 photon in one of the modes, $1-\tilde{P}$. Also, we note explicitly this step does not correspond to any procedure in our experiment, but is just an analytic tool to bound the entanglement and express it in terms of quantities that can be easily determined without too large uncertainty (unlike higher-order matrix elements such as $p_{12}$, etc).

\section{a. Detection window}

In Fig. 3, we show results for a smaller detection window that give a higher estimation for the amount of entanglement between the fields. The use of a smaller detection window can also be understood as a reduction from multiple to single modes by filtering out different temporal modes. This is a procedure that can be exactly mimicked by Alice and Bob performing that same temporal filtering on their local modes and hence can only decrease the actual entanglement (note though, that the estimate of entanglement can be increased by this procedure, even though the actual entanglement decreases. The estimated value just gets much closer to the actual value than when not filtering out extraneous modes).

For the possibly remaining multiple modes in the smaller time window, we may suppose Alice and Bob each apply the following fictitious transformation

$$
\left|n_{1}\right\rangle\left|n_{2}\right\rangle \ldots \rightarrow\left|\sum_{i} n_{i}\right\rangle\left|n_{1}, n_{2}, \ldots\right\rangle_{M}
$$

to their local modes. This transformation collects all photons in one mode (the first ket), and keeps track of where they came from in a separate system $M$ (for "memory"), such that the transformation is unitary. This transformation, therefore, leaves the entanglement between Alice's and Bob's mode unchanged. Our detectors not being able to distinguish different modes within the same time window then boils down to tracing out the memory system, which can only decrease the entanglement.

\section{Measurements}

All measurements are performed using Geiger-mode avalanche photodiodes (APDs). We assume there are only two outcomes of a photodetection measurement, corresponding to no click or some nonzero number of clicks (indeed, 
that is the only information used in the experiment). Thus, if the incoming mode $2_{L}$ is described by an annihilation operator $a$ then the photodetector performs a POVM of the form

$$
\begin{aligned}
& \Pi_{0}=\sum_{n \geq 0}(-1)^{n} \frac{a^{\dagger n} a^{n}}{n !} \\
& \Pi_{1}=I_{A}-\Pi_{0},
\end{aligned}
$$

with $I_{A}$ the identity on $2_{L}$. The corresponding probabilities are denoted by $p_{0}$ and $p_{1}$,

$$
p_{k}=\operatorname{Tr} \rho_{2_{L}} \Pi_{k},
$$

if $\rho_{2_{L}}$ is the state of mode $2_{L}$. To deduce higher-order probabilities with $k \geq 2$, beam splitters can be employed to divide and direct the mode $a$ to multiple detectors.

Joint probabilities for measurements on the two modes $2_{L}$ and $2_{R}$ can be determined in a similar way if we introduce the annihilation operator $b$ for mode $2_{R}$ and the corresponding operators $\Pi_{n}^{A, B}$ for $n=0,1$ for the two modes. The operators $\Pi_{0}$ and $\Pi_{1}$ above were written in normal order. Similarly, joint measurement probabilities can be written as

$$
P_{m n}=\operatorname{Tr} \tilde{\rho}_{2_{L}, 2_{R}}: \Pi_{m}^{A} \otimes \Pi_{n}^{B}:,
$$

where all annihilation operators $a$ and $b$ are written to the right of all creation operators $a^{\dagger}$ and $b^{\dagger}$. For example, this allows one to include easily the effects of finite efficiencies of detectors: If $\eta$ is the efficiency of a detector and $a$ the annihilation operator for the mode impinging on the detector, we may replace $a \rightarrow \sqrt{\eta} a+\sqrt{1-\eta} v$ where $v$ acts on an auxiliary mode that is assumed to be in the vacuum state. Terms with nonzero powers of $v$ then do not contribute to counting rates provided we evaluate these by using a normally-ordered expression. In that case, we can ignore $v$ and just replace $a \rightarrow \sqrt{\eta} a$. The same replacement can be used to take into account losses during propagation.

The most straightforward way to determine $\tilde{\rho}_{2_{L}, 2_{R}}$ consists of two stages. The first stage determines the diagonal elements, the second the off-diagonal elements: From the measured frequencies of joint detection events we obtain estimates for the corresponding probabilities for those joint events in terms of the underlying density matrix elements. Inverting these expressions gives estimates on the elements of the density matrix.

\section{a. Diagonal elements}

Conditioned upon detection of an event at either detector $D_{1 a}$ or $D_{1 b}$, the diagonal elements of $\tilde{\rho}_{2_{L}, 2_{R}}$ were measured by the setup described in Figure 1(c). Photons in mode $2_{L}$ are detected by a photodetector $D_{2 a}$ but mode $2_{R}$ is split on a 50/50 (approximately) beamsplitter and photons in the two resulting modes are counted by photodetectors $D_{2 b}$ and $D_{2 c}$. Starting with mode operators $a$ and $b$ for modes $2_{L}$ and $2_{R}$, there are several transformations affecting $a$ and $b$. Denoting by $a_{1}$ the mode operator detected by detector $D_{2 a}$, and by $b_{1}$ and $b_{2}$ those detected by $D_{2 b}$ and $D_{2 c}$, the transformations are simply

$$
\begin{aligned}
a_{1} & =\sqrt{\eta_{L} \eta_{1}} a \\
b_{2} & =\sqrt{\eta_{R} \eta_{2}} b / \sqrt{2} \\
b_{3} & =\sqrt{\eta_{R} \eta_{3}} b / \sqrt{2}
\end{aligned}
$$

with $\eta_{L, R}$ indicating the total efficiency of propagating to the detectors, and $\eta_{1,2,3}$ the detector efficiencies of detectors $D_{2 a}, D_{2 b}, D_{2 c}$. One then obtains expressions for the expected joint count probabilities $p_{k l m}$ with $k, l, m=0,1$ by substituting (B7) into (B4) and (B6). When acting on $\tilde{\rho}_{2_{L}, 2_{R}}$ the operators $a$ and $b$ are understood to be $a \otimes I_{B}$ and $I_{A} \otimes b$.

In the end, we only consider the total number of counts in detectors $D_{2 a}$ and $D_{2 b}, D_{2 c}$ together, leading to joint probabilities $Q_{m n}$ with $m=0,1$ and $n=0,1,2$,

$$
Q_{m n}=\sum_{m} \sum_{s+t=n} P_{m s t} .
$$

Again, for these measurements as well as those in the next section, the detection events at $D_{2 a}$ and $D_{2 b}, D_{2 c}$ are conditioned upon an event at either $D_{1 a}$ or $D_{1 b}$. This gives expressions for the expected detection probabilities $Q_{m n}$ as functions of the diagonal elements $p_{m n}$ of the density matrix: conversely, given the experimentally determined $Q_{m n}$ we invert the expressions to obtain estimates for $p_{m n}$. 


\section{b. Off-diagonal elements}

The off-diagonal elements are measured by including two extra elements: one is a phase shifter in one of the modes, say mode $2_{L}$, thus replacing $a \rightarrow \exp (i \varphi) a$. This phase is varied to produce the interference pattern ("fringes") of Figure 2. Second is an extra 50/50 beamsplitter after the phase shifter $\left[B S_{2}\right.$ in Fig. 1(d)]. One again easily arrives at simple expressions for the operators $a_{1}$ and $b_{1}, b_{2}$ detected in terms of $a$ and $b$, similar to that of (B7). Just as before, one then obtains expressions for the expected joint count probabilities $p_{k l m}$ with $k, l, m=0,1$ by substituting these expressions for $a_{1}, b_{1}, b_{2}$ into (B4) and (B6). We thus find the joint detection probabilities $Q_{m n}^{f}$ for the "fringes" as a function of $d$ and the diagonal elements of $\tilde{\rho}_{2_{L}, 2_{R}}$. Since we already obtained the diagonal elements in the previous step, we then find an estimate of the diagonal element $d$ from the visibility of the fringes.

Note that we have also carried out measurements with detector $D_{2 a}$ replaced by a beam splitter and a pair of APDs, as for detectors $D_{2 b}, D_{2 c}$. In this way, we confirm explicitly that higher order events with $k \geq 2$ at $D_{2 a}$ have a negligible impact on our estimates of fringe visibility and of the probabilities $p_{00}, p_{10}, p_{01}, p_{11}$ that enter into the determination of the concurrence $C$, in agreement with the independent assessment from $D_{2 b}, D_{2 c}$.

In addition to the above analysis we also performed a maximum likelihood analysis of the density matrix. Given the actually detected photon statistics for both measurements of diagonal and off-diagonal elements together one can, for each possible candidate density matrix $\rho_{2_{L}, 2_{R}}$, calculate the probability that the actually obtained measurement outcomes occur. Maximizing that probability over all possible density matrices gives the most likely density matrix. This estimate has been used as an additional check on the inferred values quoted in the main text and their errors.

[1] Nielsen, M. A. \& Chuang, I. L., Quantum Computation and Quantum Information, (Cambridge University Press, Cambridge, United Kingdon, 2000).

[2] For a comprehensive overview of experimental progress, see Quantum Information and Computation Roadmap, http://qist.lanl.gov/qcomp_map.shtml.

[3] Copsey, D., Oskin, M., Impens, F., Metodiev, T., Cross, A., Chong, F.T., Chuang, I.L. \& Kubiatowicz, J., Toward a scalable, silicon-based quantum computing architecture. IEEE Journal of Selected Topics in Quantum Electronics 915521569 (2003).

[4] Briegel, H.-J., Dür, W., Cirac, J.I. \& Zoller, P., Quantum Repeaters: The role of imperfect local operations in quantum communication. Phys. Rev. Lett. 81, 5932-5935 (1998).

[5] Giovannetti, G., Lloyd, S., \& Maccone, L., Quantum-enhanced measurements: beating the standard quantum limit. Science, 306, 1330-1336 (2004).

[6] Duan, L.-M., Lukin, M., Cirac, J. I. \& Zoller, P., Long-distance quantum communication with atomic ensembles and linear optics. Nature 414, 413-418 (2001).

[7] Clauser, J. F. \& Shimony, A., Bell's theorem: experimental tests and implications. Rep. Prog. Phys. 41, 1881-1927 (1978).

[8] Aspect, A., Dalibard, J. \& Roger, G., Experimental test of Bell's inequalities using time-varying analyzers. Phys. Rev. Lett. 49, 1804-1807 (1982).

[9] Optical Coherence and Quantum Optics, Mandel, L. \& Wolf, E. (Cambridge University Press, 1995$)$, Chapter 12.

[10] Haffner, H. et al., Robust entanglement. App. Phys. B 81 151-153(2005).

[11] Langer, C. et al. Long-lived qubit memory using atomic ions. Phys. Rev. Lett. 95, 060502 (2005).

[12] Julsgaard, B., Kozhekin, A. \& Polzik, E. S., Experimental long-lived entanglement of two macroscopic objects, Nature 413, 400-403 (2001).

[13] Marcikic, I., de Riedmatten, H., Tittel, W., Zbinden, H., Legré, M. \& Gisin, N., Distribution of time-bin entangled qubits over $50 \mathrm{~km}$ of optical fiber. Phys. Rev. Lett. 93, 180502 (2004).

[14] Ou, Z. Y., S. F. Pereira, Kimble, H. J. \& Peng, K. -C., Realization of the Einstein-Podolsky-Rosen paradox for continuous variables. Phys. Rev. Lett. 68, 3663-3666 (1992).

[15] Turchette, Q. A., Wood, C. S., King, B. E., Myatt, C. J., Leibfried D., Itano, W.M., Monroe, C. \& Wineland, D. J., Deterministic entanglement of two trapped ions. Phys. Rev. Lett. 81, 3631-3634 (1998).

[16] Blinov, B. B., Moehring, D. L. , Duan, L.-M. \& Monroe, C., Observation of entanglement between a single trapped atom and a single photon. Nature 428, 153-157 (2004), and references therein.

[17] Felinto, D., Chou, C.W., de Riedmatten, H., Polyakov, S.V. \& Kimble, H.J., Control of decoherence in the generation of photon pairs from atomic ensembles. Phys. Rev. A (in press, 2005); available as quant-ph/0507127

[18] Kuzmich, A., Bowen, W.P., Boozer, A.D., Boca, A., Chou, C.W., Duan, L.-M., \& Kimble, H.J., Generation of nonclassical photon pairs for scalable quantum communication with atomic ensembles. Nature 423, 731-734 (2003).

[19] van der Wal, C. H. et al., Atomic memory for correlated photon states. Science 301, 196-200 (2003).

[20] Jiang, W., Han, C., Xue, P., Duan, L.-M. \& Guo, G.-C., Nonclassical photon pairs generated from a room-temperature atomic ensemble. Phys. Rev. A 69, 043819 (2004).

[21] Chou, C.W., Polyakov, S.V., Kuzmich, A., \& Kimble, H.J., Single-photon generation from stored excitation in an atomic ensemble. Phys. Rev. Lett. 92, 213601 (2004). 
[22] Eisaman, M.D., Childress, L., André, A., Massou, F., Zibrov, A.S., \& Lukin, M.D., Shaping quantum pulses of light via coherent atomic memory. Phys. Rev. Lett. 93, 233602 (2004).

[23] Polyakov, S.V., Chou, C.W., Felinto, D. \& Kimble, H.J., Temporal dynamics of photon pairs generated by an atomic ensemble. Phys. Rev. Lett. 93, 263601 (2004).

[24] Balic, V., Braje, D.A., Kolchin, P., Yin, G.Y. \& Harris, S.E., Generation of paired photons with controllable waveforms. Phys. Rev. Lett. 94, 183601 (2005).

[25] Matsukevich, D. N., Chanelière, T., Bhattacharya, M., Lan, S.-Y., Jenkins, S. D., Kennedy, T. A. B. \& Kuzmich, A., Entanglement of a Photon and a Collective Atomic Excitation. Phys. Rev. Lett. 95, 040405 (2005).

[26] Matsukevich, D.N. \& Kuzmich, A., Quantum state transfer between matter and light. Science 306, 663-666 (2004).

[27] van Enk, S. \& Kimble, H. J., Comment on "Quantum state transfer between matter and light." Science, 309, 1187b (2005).

[28] Matsukevich, D. N. \& Kuzmich, A., Response to Comment on "Quantum state transfer between matter and light." Science, 309, 1187c (2005).

[29] Duan, L.-M., Cirac, J.I. \& Zoller, P., Three-dimensional theory for interaction between atomic ensembles and free-space light. Phys. Rev. A 66, 023818 (2002).

[30] Wootters, W. K., Entanglement of formation of an arbitrary state of two qubits. Phys. Rev. Lett. 80, 2245-2248 (1998).

[31] C.H. Bennett, D.P. DiVincenzo, J.A. Smolin, and W. Wootters, Phys. Rev. A 54, 3824 (1996). 